\title{
Symmetry of the Top Valence Band States in GaN/AlGaN Quantum Wells and its Influence on the Polarization of Emitted Light
}

\author{
W. BARDYSZEWSKI ${ }^{a}$ AND S.P. ŁEPKOWSKI ${ }^{b}$ \\ ${ }^{a}$ Institute of Theoretical Physics, Faculty of Physics, University of Warsaw, Hoża 69, 00-681 Warszawa, Poland \\ ${ }^{b}$ Institute of High Pressure Physics, "Unipress", Polish Academy of Sciences \\ Sokołowska 29/37, 01-142 Warszawa, Poland
}

\begin{abstract}
We show theoretically that for narrow GaN/AlGaN quantum wells, lattice matched to GaN substrate/buffer and grown along the (0001) crystallographic direction the topmost valence subband symmetry depends critically on such parameters as quantum well thickness and barrier composition. This effect determines polarization of the emitted light. It is noted that the symmetry of the topmost valence band level is sensitive to the values of the $D_{3}$ and $D_{4}$ deformation potentials and can be employed in verification of existing literature values of these parameters.
\end{abstract}

PACS: 78.40.Fy, 78.20.Bh, 78.30.Fs

\section{Introduction}

Group III-N semiconductors crystallizing in the wurtzite structure have complex valence band structure in the vicinity of the $\Gamma$ point consisting of three subbands corresponding to the heavy hole (HH) subband with symmetry $\Gamma_{9}$, light hole $(\mathrm{LH})$ and the crystal-field split-off subbands $(\mathrm{CH})$ built of functions of symmetry $\Gamma_{7}$. In the unstrained bulk GaN and $\mathrm{InN}$ the top subband is of $\mathrm{HH}$ character whereas in the bulk AlN the sequence of subbands is inverted with topmost light hole subband. This inversion obviously is present in AlGaN alloys. Combining two materials such as AlGaN and GaN in one quantum well (QW) system gives an opportunity to modify the structure of the top of the valence band states, especially in the presence of strong electric field (introduced by piezoelectric and spontaneous polarizations) which effectively mixes the well and barrier states. Quantum structures built with these semiconductor materials, conventionally grown along the polar (0001) crystallographic direction of the wurtzite structure, are used to produce ultraviolet light emitters and high power electronic devices [1]. In fact, in most cases the character of the topmost valence subband in GaN/AlGaN QWs is usually of HH type. However in very narrow QWs a reordering of the top valence subbands may occur resulting in the $\mathrm{LH}$ subband at the top of the valence band. It was established by Shields et al. that this effect was responsible for the significant change of the excitonic $g$-factor with the well width [2].

The description of this problem requires taking into account the large biaxial strains and huge built-in electric fields which are present in these structures and lead to dramatic changes in the electronic states of the conduction and valence bands.

We provide the quantitative model of this phenomenon in the framework of the $k \cdot p$ method demonstrating how sensitive is the ordering of the topmost valence levels to the exact values of relevant parameters, in particular the deformation potentials $D_{3}$ and $D_{4}$ [3]. In order to illustrate the effect of reordering of the topmost valence subbands on polarization of emitted light, we present theoretical excitonic absorption and emission spectra for various $\mathrm{QW}$ structures.

\section{Model}

Using the linear theory of elasticity and piezoelectricity we determine the strain and the built-in electric field the QW introduced by the lattice mismatch between the QW and the barrier. The effective one-particle Hamiltonian for the QW including the influence of strain and the electric field due to the spontaneous polarization and piezoelectric effect is then diagonalized in order to obtain one electron spectra in the valence and conduction subbands. The optical spectra are obtained by solving the Bethe-Salpeter exciton equation in the envelope function representation using the Landau orbitals basis set to describe the relative electron-hole motion. The interband electron-hole excitations are represented by the correlation function $\Psi_{1,2}:=\Theta(t)\left\langle\left[a_{2}^{+}(t) a_{1}(t), \hat{P}_{\varepsilon}^{\dagger}(0)\right]\right\rangle$ where $a_{1}^{+}$and $a_{2}$ are creation and annihilation operators for electron band states 1 and 2 , respectively. The momentum operator has the form $\hat{P}_{\varepsilon}^{\dagger}(t)=\sum_{1,2} P_{1,2}^{\varepsilon} a_{1}^{+}(t) a_{2}(t)$, where $P_{1,2}^{\varepsilon}=\left\langle 1\left|\hat{p}_{\varepsilon}\right| 2\right\rangle$ is equal to the matrix element of 
$\hat{p}_{\varepsilon}$ - the projection of the one-particle momentum operator onto the light polarization vector $\varepsilon$. Here we use the convention in which the indices 1 denote single electron states in the QW conduction subbands and the indices 2 denote the states in the valence subbands.

The exciton equation takes the form of an effective Schrödinger equation

$$
\begin{aligned}
& \mathrm{i} \frac{\partial}{\partial t} \Psi_{1,2}(t)=\sum_{\overline{1}, \overline{2}}\left(H_{1, \overline{1}}^{\mathrm{c}} \delta_{2, \overline{2}}-H_{2, \overline{2}}^{\mathrm{v}} \delta_{1, \overline{1}}\right) \Psi_{\overline{1}, \overline{2}}(t) \\
& \quad-\sum_{\overline{2}, \overline{1}} V_{1, \overline{2}, 2, \overline{1}} \Psi_{\overline{1}, \overline{2}}(t)+\mathrm{i} \delta(t) P_{1,2}^{\varepsilon} .
\end{aligned}
$$

The single-particle Hamiltonian operator $H_{1, \overline{1}}^{\mathrm{c}}$ describes the conduction band states in wurtzite QWs in the parabolic band approximation. The valence band term $H_{2, \overline{2}}^{\mathrm{v}}$ is equal to the transposed Rashba-ShekaPikus Hamiltonian matrix for wurtzite QWs [4] while the electron-hole Coulomb interaction screened by the static dielectric constant is denoted by $V_{1, \overline{2}, 2, \overline{1}}$. The exciton equation is solved using the eigenvector expansion method. According to the linear-response theory the absorption coefficient can be written as

$$
\begin{aligned}
& \alpha(\omega)=\frac{4 \pi m_{0} e^{2}}{c n_{\mathrm{r}} \hbar^{2} \omega \Omega} \\
& \quad \times \operatorname{Re} \int_{0}^{\infty}\left\langle\left[\hat{P}_{\varepsilon}(t), \hat{P}_{\varepsilon}^{+}(0)\right]\right\rangle \mathrm{e}^{\mathrm{i}\left(\omega+\mathrm{i} 0^{+}\right) t} \mathrm{~d} t,
\end{aligned}
$$

where $\Omega$ is the system volume, $n_{\mathrm{r}}$ - the refractive index, $m_{0}$ - electron mass, $e$ - elementary charge, and the angular brackets represent ensemble averaging. In calculation of optical spectra, we have assumed a Gaussian shape for each transition line with the standard FWHM equal to $8 \mathrm{meV}$. The emission spectra are evaluated from absorption curves using the Einstein relation and assuming the temperature of $80 \mathrm{~K}$.

\section{Results and discussion}

We demonstrate first the dependence of the valence band energy levels of the $\mathrm{GaN} / \mathrm{Al}_{x} \mathrm{Ga}_{1-x} \mathrm{~N}$ QWs lattice matched to the GaN substrate on the well width assuming constant width of barriers.

In our calculations we used the compilation of band parameters given in Refs. [5-7]. The deformation potentials for the valence band obtained by using the density functional method with the hybrid Heyd-Scuseria-Ernzerhof functional are taken from the recent publication [3]. The parameters for $\mathrm{Al}_{x} \mathrm{Ga}_{1-x} \mathrm{~N}$ are estimated using the linear interpolation between binaries except for the energy gap and the spontaneous polarization for which bowing is taken into account as in Ref. [6]. The diagram in Fig. 1 shows the dominant, $\Gamma_{7}$ (stars) or $\Gamma_{9}$ (squares), character of the topmost valence subband depending on the $\mathrm{Al}$ content in the barrier and $\mathrm{QW}$ width in $\mathrm{GaN} /$ $\mathrm{Al}_{x} \mathrm{Ga}_{1-x} \mathrm{~N}$ multiquantum wells system for fixed barrier thickness equal to $40 \mathrm{~nm}$. While for narrow QWs, one can

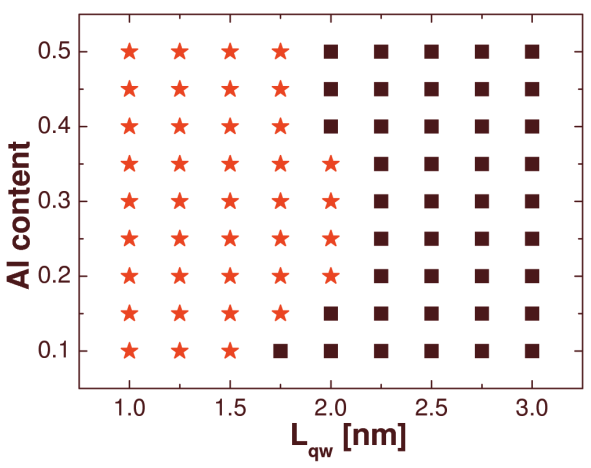

Fig. 1. Dominant character of the uppermost valence subband for $\mathrm{GaN} / \mathrm{Al}_{x} \mathrm{Ga}_{1-x} \mathrm{~N}$ QWs with barrier width equal to $40 \mathrm{~nm}$. Stars correspond to $\Gamma_{7}$ and squares to $\Gamma_{9}$ symmetry of the uppermost subband, respectively. Results have been obtained using the deformation potentials $D_{3}$ and $D_{4}$ taken from Ref. [3].

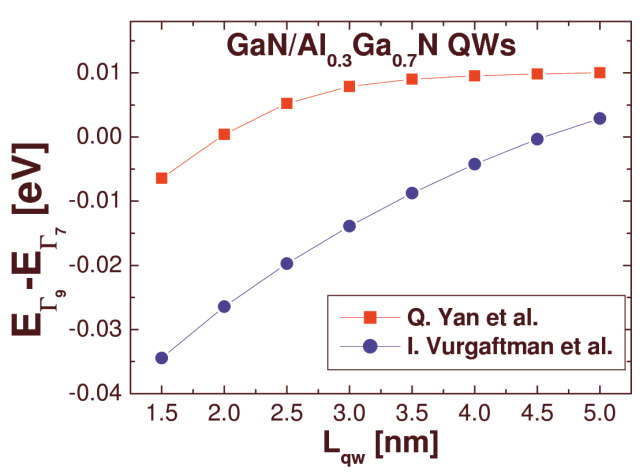

Fig. 2. Energy difference of two top most valence subband levels in $\mathrm{GaN} / \mathrm{Al}_{0.3} \mathrm{Ga}_{0.7} \mathrm{~N}$ QWs with barrier width equal to $40 \mathrm{~nm}$ as a function of $\mathrm{QW}$ width. Squares and circles correspond to the results obtained using the deformation potentials $D_{3}$ and $D_{4}$ taken from Ref. [3] and Ref. [5], respectively.

observe that the topmost valence subband is of $\Gamma_{7}$ character, with increase of the QW width, reordering of $\Gamma_{7}$ and $\Gamma_{9}$ valence band levels occurs. The critical well width corresponding to subband reordering, is the largest for the $\mathrm{Al}$ content in the barrier ranging from $x=0.2$ to $x=0.35$. This critical well width becomes much larger if the deformation potentials $D_{3}$ and $D_{4}$ from Ref. [5] are used instead. This effect is illustrated by Fig. 2 which shows the energy difference of two topmost valence subband levels in $\mathrm{GaN} / \mathrm{Al}_{0.3} \mathrm{Ga}_{0.7} \mathrm{~N}$ QWs with barrier width equal to $40 \mathrm{~nm}$ as a function of $\mathrm{QW}$ width. The band reordering for deformation potentials from Ref. [5] occurs for much wider QWs than that in the case of Ref. [3].

In order to illustrate the effect of band reordering on the optical properties of $\mathrm{GaN} / \mathrm{Al}_{x} \mathrm{Ga}_{1-x} \mathrm{~N}$ multiquantum wells systems, we present in Figs. 3 and 4, respectively, the calculated excitonic absorption and photoluminescence spectra in QWs with aluminum content in the barrier $x=0.3$ and well widths equal to $1,2,3$ and $4 \mathrm{~nm}$ 


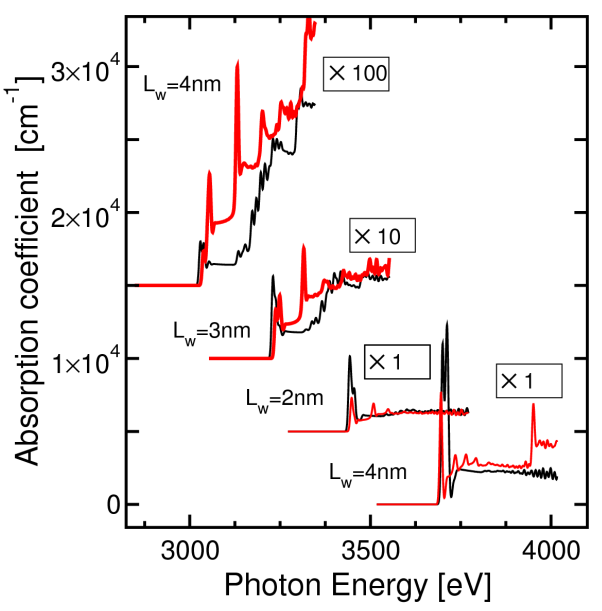

Fig. 3. Absorption spectra in $\mathrm{GaN} / \mathrm{Al}_{0.3} \mathrm{Ga}_{0.7} \mathrm{~N}$ QWs with widths $1,2,3$ and $4 \mathrm{~nm}$ for circular polarization of light in the quantum well plane (black lines) and linear, $z$-polarization, perpendicular to the QW plane (red curves). Let us note that the results for the $3 \mathrm{~nm}$ wide QW were multiplied by a factor of 10 while the results for the $4 \mathrm{~nm}$ wide QW were multiplied by 100 .

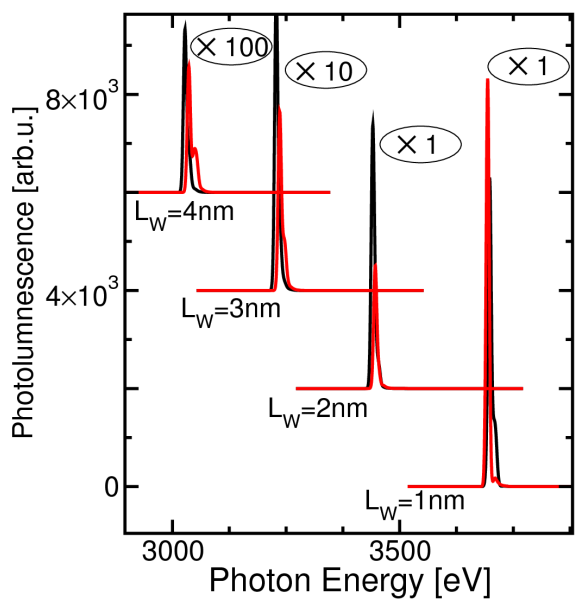

Fig. 4. Calculated photoluminescence spectra for $\mathrm{GaN} / \mathrm{Al}_{0.2} \mathrm{Ga}_{0.8} \mathrm{~N}$ QWs with widths $1,2,3$ and $4 \mathrm{~nm}$ for circular polarization of light in the QW plane (black lines) and linear, $z$-polarization perpendicular to the QW plane (red curves). Let us note that the results for the $3 \mathrm{~nm}$ wide QW were multiplied by a factor of 10 while the results for the $4 \mathrm{~nm}$ wide QW are multiplied by 100 .

for circular polarization of light in the QW plane (black lines) and linear, $z$-polarization, perpendicular to the
QW plane (red curves). Clearly the fundamental excitonic transition with polarization along $z$-axis (associated with $\Gamma_{7}$ symmetry levels) has lower energy than the transition with circular polarization from $\Gamma_{9}$ levels in the $4 \mathrm{~nm}$ wide QW. The opposite occurs for a narrow, $1 \mathrm{~nm}$ wide QW.

\section{Conclusions}

Within the framework of the continuum theory of elasticity and piezoelectricity, and $\boldsymbol{k} \cdot \boldsymbol{p}$ method with the Rashba-Sheka-Pikus valence band Hamiltonian applied to description of QW states in the envelope function approximation we have shown that for sufficiently narrow quantum wells the topmost valence subband is built from $\Gamma_{7}$-like wave functions. As a consequence the fundamental optical transitions occur only with polarization of light parallel to the $z$-axis (perpendicular to the QW plane) in such systems. For wider wells, the topmost valence subband originates from the $\Gamma_{9}$ bulk states which allow for transitions with polarization of light in-plane of the QW. The critical value of the QW for which the selection rules are changing is very sensitive to the values of deformation potentials $D_{3}$ and $D_{4}$ which unfortunately are not known. We believe that experimental studies of polarization of light emitted from $\mathrm{GaN} / \mathrm{Al}_{x} \mathrm{Ga}_{1-x} \mathrm{~N}$ QW systems may lead to better determination of those potentials.

\section{Acknowledgments}

This work was supported by the Polish State Committee for Scientific Research, project No. NN202 010134.

\section{References}

[1] H. Morkoc, Nitride Semiconductors and Devices, Springer-Verlag, Berlin 1999.

[2] P.A. Shields, R.J. Nicholas, N. Grandjean, J. Massies, Phys. Rev. B 63, 245319 (2001).

[3] Q. Yan, P. Rinke, M. Scheffler, C.G.V. de Walle, Appl. Phys. Lett. 95, 121111 (2009).

[4] S.L. Chuang, C.S. Chang, Phys. Rev. B 54, 2491 (1996).

[5] I. Vurgaftman, R. Meyer, J. Appl. Phys. 94, 3675 (2003).

[6] S.P. Łepkowski, J.A. Majewski, G. Jurczak, Phys. Rev. B 72, 245201 (2005).

[7] P. Rinke, M. Winkelnkemper, A. Qteish, D. Bimberg, J. Neugebauer, M. Scheffler, Phys. Rev. B 77, 075202 (2008). 